\title{
Predisposing Hemodynamic Factors Associated with a Failed Apnea Test during Brain Death Determination
}

\author{
Eun Young Kim, M.D., and Ji Hyun Kim, M.D. \\ Division of Trauma and Surgical Critical Care, Department of General Surgery, Seoul St. Mary's Hospital, The Catholic University of Korea College of Medicine, Seoul, Korea
}

Background: The apnea test is an essential component in the clinical determination of brain death, however it may incur a significant risk of complications such as hypotension, hypoxia and even cardiac arrest. We analyzed the risk factors associated with a failed apnea test during brain death assessment in order to predict and avoid these adverse events.

Methods: Medical records on apnea tests performed for brain-dead donors at our institution between January 2009 and January 2016 were retrospectively reviewed. Age, gender, etiology of brain death, use of catecholamines and results of arterial blood gas analysis (ABGA), systolic/diastolic blood pressure (SBP/DBP), mean arterial pressure and central venous pressure prior to apnea test initiation were collected as variables. A-a gradient and $\mathrm{P}_{\mathrm{aO} 2} / \mathrm{F}_{\mathrm{iO2}}$ were calculated for more precise assessment of the respiratory system. In total, 267 cases were divided into two groups based on those who completed the apnea test and those who failed the test.

Results: 13 cases failed the apnea test. Among them, seven cases failed due to severe hypotension ( $\mathrm{SBP}<60 \mathrm{mmHg}$ ) and the others failed due to refractory hypoxia. In terms of hemodynamic state, SBP was significantly higher in the completed test group than the failed group (126.5 \pm 23.9 vs. $103 \pm 15.2$, respectively; $p=0.001)$. In $A B G A$, the completed test group showed significantly higher $P_{a 02} /$ $\mathrm{F}_{\mathrm{iO} 2}(313.6 \pm 229.8$ vs. $141.5 \pm 131.0$, respectively; $\mathrm{p}=0.008)$ and a lower $A$-a gradient $(278.2 \pm 209.5$ vs. $506.2 \pm 173.1$, respectively; $p=$ $0.000)$. In multivariable analysis, low SBP $(p=0.003)$ and high A-a gradient $(p=0.044)$ were independent risk factors associated with a failed apnea test.

Conclusions: Although the unexpected adverse events during the apnea test for brain death determination do not occur frequently, they can be fatal. If a brain-dead patient has low SBP and a high A-a gradient, clinicians should pay more attention and prepare for potential complications prior to the apnea test.

Key Words: apnea test; brain death; predisposing factor.

\section{Introduction}

Brain death is defined as the irreversible loss of all brain functions in the presence of definite coma and absence of brainstem reflexes. Brain death usually has a specific, identifiable cause, excluding hypoxic brain injury resulting from drug or alcohol intoxication, hypothermia and cardiac arrest.[1,2] Most transplants that use organs retrieved from brain

Received on April 25, 2016 Revised on May 23, 2016 Accepted on May 27, 2016

Correspondence to: Ji Hyun Kim, Department of Surgery, Seoul St. Mary's Hospital, The Catholic University of Korea College of Medicine, 222 Banpo-daero, Seocho-gu, Seoul, 06591, Korea

Tel: +82-2-2258-2876, Fax: +82-2-595-2822

E-mail: doctor2003@catholic.ac.kr

*No potential conflict of interest relevant to this article was reported. dead patients can be the best treatment option for a wide range of patients with end stage organ failure, when their conditions may not be improved through other clinical practices. However, the persistent shortage of donor organs continues as the number of patients waiting for organ transplants far exceeds the number of donor organs available. To reduce this problem, it is important to provide 
adequate management and decision-making for potential brain dead organ donors, maximize the number of donor organs through early monitoring of hemodynamic changes and aggressive treatment and preserve subsequent function of organs procured.

To diagnose brain death, the accuracy of clinical evaluation in determining the cause of irreversible brain injury is prerequisite prior to a series of examinations required to determine brain function such as brainstem reflexes and respiratory center function. Among essential tests to determine brain death, apnea test is designed to detect the loss of respiratory control centers in the brainstem, which is identified through the absence of respiratory reflexes in response to hypercapnia induced by temporary arrest of ventilation in potentially brain dead donors. Although apnea test is safely conducted in most cases, a sudden discontinued ventilation may result in extensive alveolar collapse and hypoxemia, and these acute respiratory problems can cause both common and rare side effects, including hypotension, cardiac arrhythmia, other cardiovascular dysfunctions and even cardiac arrest and pneumothorax.[3,4] Further hemodynamic deterioration can cause hypoperfusion and organ failure, forcing the failure of apnea test and making organ donation impossible. Risk factors for apnea test failure have been discussed in several small scale studies.[1,5] However, criteria for associated risks have not been clearly established.

In this study, the authors investigated hemodynamic data and lab results of potential brain dead donors who required an apnea test to confirm brain death at a tertiary hospital to identify risk factors associated with apnea test failure.

\section{Materials and Methods}

This study was conducted at a tertiary hospital in Korea during the period January 2009-January 2016. Sample size was 267 patients who developed irreversible brain injury and received an apnea test for the purpose of organ donation, excluding those who had hypoxic brain dam- age resulting from drug or alcohol intoxication, hypothermia and cardiac arrest Subjects were divided into two groups based on success or failure of apnea test: The success group completed an apnea test and the failure group could not complete the test because of death and other reasons. We conducted analysis on age, gender, cause of brain death, use of vasopressors such as catecholamine, arterial blood gas analysis (ABGA) results, systolic and diastolic blood pressure just before apnea test, mean arterial pressure and central venous pressure to compare the two groups. To analyze respiratory status and lung function, the $\mathrm{P}_{\mathrm{aO} 2} / \mathrm{F}_{\mathrm{iO} 2}$ ratio was measured and alveolar-arterial gradient (A-a gradient) was calculated using the equation suggested in the Mellemgaard's study:[6]

$$
\begin{aligned}
& \text { A-a gradient }=\mathrm{P}_{\mathrm{AO} 2}-\mathrm{P}_{\mathrm{aO} 2}, \mathrm{P}_{\mathrm{AO} 2}=\left(\mathrm{F}_{\mathrm{iO} 2} \times\left[\mathrm{P}_{\mathrm{atm}}-\mathrm{P}_{\mathrm{H} 2 \mathrm{O}}\right]\right) \\
& -\left(\mathrm{P}_{\mathrm{aCO} 2} \div 0.8\right)
\end{aligned}
$$

where $\mathrm{P}_{\mathrm{AO} 2}$ is partial pressure of oxygen dissolved in the pulmonary alveoli; $\mathrm{P}_{\mathrm{atm}}$ is barometric pressure $(760$ $\mathrm{mmHg}) ; \mathrm{P}_{\mathrm{H} 20}$ is partial pressure of water $(47 \mathrm{mmHg})$ at temperature of $37^{\circ} \mathrm{C}$; and $\mathrm{P}_{\mathrm{aCO} 2}$ is partial pressure of carbon dioxide in arterial blood. This study is a retrospective study involving collection and analysis of medical record information and received approval from the Institutional Review Board of Seoul St. Mary's Hospital.

All subjects were intubated through the endotracheal route and receiving mechanical ventilation (MV) support, and their blood pressure was constantly monitored via the catheter inserted into the radial artery. Subjects received fluid substances and medication through a central venous catheter. Before apnea testing, blood was collected from the radial artery to determine standard $\mathrm{P}_{\mathrm{aCO} 2}$ and the fraction of inspired oxygen $\left(\mathrm{F}_{\mathrm{iO} 2}\right)$ on $\mathrm{MV}$ was increased to 1.0 to achieve the process of pre-oxygenation for 10 minutes. After the pre-oxygenation period, MV was discontinued and apnea test was performed by pushing the endotracheal tube close to the carina and administering oxygen of $6 \mathrm{~L}$. All subjects were observed for signs of spontaneous breathing or chest or abdominal movements for up to 10 minutes. During this observation period, arterial blood 
samples were collected through a catheter placed in the radial artery to perform ABGA. If signs of spontaneous breathing/chest or abdominal movements are absent and the $\mathrm{P}_{\mathrm{aCO} 2}$ is $\geq 60 \mathrm{mmHg}$, we concluded that the apnea test is passed. However, if breathing effort is observed with severe hypotension (systolic blood pressure less than 60 $\mathrm{mmHg}$ ) despite administration of fluids and vasopressors, $\mathrm{P}_{\mathrm{aCO} 2}$ of less than $60 \mathrm{mmHg}, \mathrm{SpO} 2$ (less than $85 \%$ ) for more than 1 minute, we aborted the apnea test and concluded that the apnea is failed.

SPSS software version 21.0 (SPSS Inc., Chicago, IL, USA) was used for statistical analysis. The $p$ value of less than 0.05 was considered statistically significant. Fisher's exact test was performed for categorical variables, and data were expressed as frequency and percentages. For continuous variables, student's $t$-test was performed, and data were expressed as mean \pm standard deviation. Risk factors associated with apnea test failure were identified on multivariate logistic regression analysis after multicollinearity was ignored in linear regression method. The $95 \%$ confidence interval was used to estimate the odds ratio.

\section{Results}

The mean age of 267 potential brain dead donors was 46.9 years (range 18-69 years). One hundred and sixty six patients $(62.2 \%)$ were male and 101 patients $(37.8 \%)$ were female. In 254 (95.1\%) of 267 subjects, an apnea test could be completed. However, in 13 patients $(4.9 \%)$, an apnea test had to be aborted or failed due to progressive conditions and death, making a clinical determination of brain death impossible. Clinical characteristics of 13 subjects with aborted apnea tests are presented in Table 1. The apnea test had to be aborted in 6 of them due to progressive hypotension and subsequent hemodynamic instability and in the remaining 7 patients due to severe refractory hypoxemia occurred after sustained $\mathrm{SpO} 2$ of less than $85 \%$.

In Table 2, the success and failure groups were compared with respect to clinical characteristics, and pre-apnea hemodynamics and ABGA results. There was no significant difference in mean age, gender and cause of brain death between the two groups, and the leading cause of brain death was subarachnoid hemorrhage in both groups

Table 1. Clinical characteristics of patient who failed the apnea test

\begin{tabular}{|c|c|c|c|c|c|c|c|c|c|}
\hline \multirow{2}{*}{ Patient } & \multirow{2}{*}{ Age/sex } & \multirow{2}{*}{$\begin{array}{l}\text { Reason for } \\
\text { failure }\end{array}$} & \multicolumn{2}{|c|}{$\begin{array}{c}\text { Pre-apnea testing } \\
\text { hemodynamic parameters }\end{array}$} & \multicolumn{5}{|c|}{ Pre-apnea testing respiratory parameters } \\
\hline & & & $\begin{array}{l}\text { Baseline SBP } \\
(\mathrm{mmHg})\end{array}$ & $\begin{array}{l}\text { Baseline DBP } \\
(\mathrm{mmHg})\end{array}$ & $\mathrm{pH}$ & $\mathrm{P}_{\mathrm{a} 02}(\mathrm{mmHg})$ & $\begin{array}{c}\mathrm{P}_{\mathrm{ac02}} \\
(\mathrm{mmHg})\end{array}$ & $\mathrm{P}_{\mathrm{a} 02} / \mathrm{F}_{\mathrm{i} 02}$ & A-a gradient \\
\hline 1 & $18 / M$ & Hypoxemia & 110 & 71 & 7.284 & 85 & 54 & 85 & 585.34 \\
\hline 2 & $36 / \mathrm{M}$ & Hypoxemia & 103 & 48 & 7.369 & 64 & 31 & 64 & 623.78 \\
\hline 3 & $38 / F$ & Hypotension & 95 & 61 & 7.373 & 150 & 29 & 300 & 183.38 \\
\hline 4 & $25 / F$ & Hypoxemia & 111 & 63 & 7.175 & 76 & 48 & 76 & 598.50 \\
\hline 5 & $31 / \mathrm{M}$ & Hypotension & 91 & 63 & 7.219 & 86 & 38 & 86 & 596.52 \\
\hline 6 & 38 / M & Hypoxemia & 122 & 82 & 7.311 & 79 & 38 & 88 & 532.26 \\
\hline 7 & 38 / M & Hypotension & 102 & 88 & 7.314 & 98 & 37 & 98 & 585.20 \\
\hline 8 & $49 / F$ & Hypoxemia & 110 & 80 & 7.353 & 74 & 31 & 74 & 613.66 \\
\hline 9 & $49 / F$ & Hypoxemia & 120 & 90 & 7.310 & 70 & 38 & 70 & 611.70 \\
\hline 10 & $52 / F$ & Hypotension & 97 & 79 & 7.265 & 406 & 30 & 508 & 140.80 \\
\hline 11 & 66 / M & Hypotension & 88 & 62 & 7.218 & 94 & 43 & 94 & 584.48 \\
\hline 12 & 50 / M & Hypoxemia & 97 & 56 & 7.286 & 67 & 42 & 67 & 613.12 \\
\hline 13 & $69 / M$ & Hypotension & 93 & 85 & 7.326 & 161 & 34 & 230 & 311.22 \\
\hline
\end{tabular}

SBP: systolic blood pressure; DBP: diastolic blood pressure; $\mathrm{P}_{\mathrm{a} 02}$ : arterial partial pressure of oxygen; $\mathrm{P}_{\mathrm{aco2}}$ : arterial partial pressure of carbon dioxide; $\mathrm{P}_{\mathrm{a} 02} / \mathrm{F}_{\mathrm{i} 02}$ : arterial partial pressure of oxygen/fraction of inspired oxygen; A-a gradient: alveolar-arterial gradient; M: male; F: female. 
(83 cases, $32.7 \%$ in success group vs. 6 cases, $46.2 \%$, in failure group, respectively; $p=0.369$ ). In terms of hemodynamics, the mean systolic blood pressure measured before an apnea test was $126.5 \pm 23.9 \mathrm{mmHg}$ in the success group and $103 \pm 15.2 \mathrm{mmHg}$ in the failure grou8p, showing a statistically significant difference $(p=0.001)$. However, there were no significant differences between the two groups with respect to the mean diastolic blood pressure $(78.5 \pm 19.1$ vs. $71.4 \pm 26.6 \mathrm{mmHg}, \mathrm{p}=0.360)$, use of catecholamine (190 cases, $74.8 \%$ vs. 12 cases, $92.3 \%$, $\mathrm{p}=0.198)$. The comparison of respiratory status between the two groups on the basis of results of standard ABGA revealed no significance difference in the mean $\mathrm{P}_{\mathrm{aCO} 2}(35.3$ \pm 4.0 vs. $37.9 \pm 7.4 \mathrm{mmHg}, \mathrm{p}=0.233)$. However, the mean $\mathrm{pH}(7.362 \pm 0.1$ vs. $7.292 \pm 0.1, \mathrm{p}=0.005)$ and $\mathrm{P}_{\mathrm{O} 2}$ $(188.4 \pm 111.8$ vs.116.2 $\pm 92.2 \mathrm{mmHg}, \mathrm{p}=0.002)$ were significantly greater in the success group, compared with the failure group. The mean $\mathrm{P}_{\mathrm{aO} 2} / \mathrm{F}_{\mathrm{iO2} 2}(313.6 \pm 229.8$ vs. $141.5 \pm 131.0, p=0.008)$ also significantly greater, and the mean a-a gradient $(278.2 \pm 209.5$ vs. $506.2 \pm 173.1$, $\mathrm{p}=0.000$ ) was significantly lower in the success group. To identify risk factors for apnea test failed or aborted, multivariate analysis was performed on the variables found to be significantly different in the two groups. To reduce the correlation between variables, the collinearity among the variables used in the linear regression was assessed, and pre-apnea systolic blood pressure, $\mathrm{pH}$ and $\mathrm{P}_{\mathrm{O} 2}$ (based on results of standard ABGA) and a-a gradient were finally used in the multiple regression analysis. the $\mathrm{P}_{\mathrm{aO} 2} / \mathrm{F}_{\mathrm{iO} 2}$ ratio was not included. As presented in Table 3 , arterial $\mathrm{pH}$ levels and $\mathrm{P}_{\mathrm{O} 2}$ were found to be not significant. However, low systolic blood pressure (Odds ratio

Table 2. Clinical characteristics and pre-test states of patients

\begin{tabular}{|c|c|c|c|c|}
\hline Variable & Total $(n=267)$ & Success $(n=254)$ & Fail $(n=13)$ & $\mathrm{p}$-value \\
\hline Age (yr) & $46.9 \pm 11.9$ & $47.1 \pm 11.7$ & $43.0 \pm 14.7$ & 0.224 \\
\hline Gender (M/F) & 166/101 & $158 / 96$ & $8 / 5$ & 1.000 \\
\hline Etiology & & & & 0.819 \\
\hline Traumatic brain injury & $71(26.6)$ & $69(27.2)$ & $2(15.4)$ & 0.524 \\
\hline ICH or IVH & $40(15.0)$ & $38(15.0)$ & $2(15.4)$ & 1.000 \\
\hline SAH & 89 (33.3) & $83(32.7)$ & $6(46.2)$ & 0.369 \\
\hline Ischemic stroke & $15(5.6)$ & $15(5.9)$ & 0 & 1.000 \\
\hline Hypoxic injury & $48(18)$ & $45(17.7)$ & $3(23.1)$ & 0.709 \\
\hline Miscellaneous & $4(1.5)$ & $4(1.6)$ & 0 & 1.000 \\
\hline \multicolumn{5}{|c|}{ Pre-apnea testing hemodynamic parameters } \\
\hline Pretesting SBP (mmHg) & $125.4 \pm 24.1$ & $126.5 \pm 23.9$ & $103 \pm 15.2$ & $0.001^{*}$ \\
\hline Pretesting DBP (mmHg) & $78.1 \pm 19.4$ & $78.5 \pm 19.1$ & $71.4 \pm 26.6$ & 0.360 \\
\hline Pretesting MAP (mmHg) & $94.0 \pm 20.0$ & $94.5 \pm 19.5$ & $84.2 \pm 26.7$ & 0.068 \\
\hline Pretesting CVP & $8.5 \pm 3.9$ & $8.4 \pm 4.0$ & $10.4 \pm 2.7$ & 0.077 \\
\hline Usage of catecholamine & $202(75.7)$ & $190(74.8)$ & $12(92.3)$ & 0.198 \\
\hline \multicolumn{5}{|c|}{ Pre-apnea testing respiratory measurements } \\
\hline $\mathrm{pH}$ & $7.359 \pm 0.9$ & $7.362 \pm 0.1$ & $7.292 \pm 0.1$ & $0.005^{*}$ \\
\hline $\mathrm{P}_{\mathrm{a} 02}(\mathrm{mmHg})$ & $185.0 \pm 111.8$ & $188.4 \pm 111.8$ & $116.2 \pm 92.2$ & $0.002^{*}$ \\
\hline $\mathrm{P}_{\mathrm{aCO} 2}(\mathrm{mmHg})$ & $35.5 \pm 4.2$ & $35.3 \pm 4.0$ & $37.9 \pm 7.4$ & 0.233 \\
\hline $\mathrm{P}_{\mathrm{a} 02} / \mathrm{F}_{\mathrm{i} 02}$ & $305.2 \pm 228.9$ & $313.6 \pm 229.8$ & $141.5 \pm 131.0$ & $0.008^{*}$ \\
\hline A-a gradient & $289.3 \pm 213.3$ & $278.2 \pm 209.5$ & $506.2 \pm 173.1$ & $0.000^{*}$ \\
\hline
\end{tabular}

M: male; F: female; ICH: intracerebral hemorrhage; IVH: intraventricular hemorrhage; SAH: subarachnoid hemorrhage; SBP: systolic blood pressure; DBP: diastolic blood pressure; MAP: mean arterial pressure; CVP: central venous pressure; $\mathrm{P}_{\mathrm{a} 02}$ : arterial partial pressure of oxygen; $\mathrm{P}_{\mathrm{aco2}}$ : arterial partial pressure of carbon dioxide; $\mathrm{P}_{\mathrm{a} 02} / \mathrm{F}_{\mathrm{i} 02}$ : arterial partial pressure of oxygen/fraction of inspired oxygen; A-a gradient: alveolar-arterial gradient. 
Table 3. Risk factors associated with failed apnea test

\begin{tabular}{lccc}
\hline Variable & 0dds ratio & $95 \%$ Cl & $p$-value \\
\hline SBP $(\mathrm{mmHg})$ & 0.949 & $0.916-0.983$ & $0.003^{*}$ \\
$\mathrm{pH}$ & 0.024 & $0.000-8.007$ & 0.208 \\
$\mathrm{P}_{\mathrm{a02}}(\mathrm{mmHg})$ & 0.994 & $0.983-1.005$ & 0.310 \\
A-a gradient & 1.005 & $1.001-1.009$ & $0.044^{*}$ \\
\hline
\end{tabular}

Cl: confidence interval; SBP: systolic blood pressure; $\mathrm{P}_{\mathrm{a} 02}$ : arterial partial pressure of oxygen; A-a gradient: alveolar-arterial gradient. ${ }^{*} p<0.05$.

$0.949,95 \%$ confidence interval $[\mathrm{CI}] 0.916-0.983, \mathrm{p}=$ 0.003 ) and elevated a-a gradient (Odds ratio 1.005, 95\% CI 1.001-1.009, $\mathrm{p}=0.044$ ) were found to be significant risk factors associated with apnea test failure.

\section{Discussion}

When brain death occurs due to irreversible brain injury including the loss of brainstem reflexes, apnea is usually diagnosed on the basis of the extensive loss of all functions of the brain, including brainstem, and absence of responses to increasing amounts of carbon dioxide in the blood.[7] Apnea test is performed to ensure these specific characteristics of apnea and deemed as the most fundamental component of diagnosis of brain death in potential organ donors. During apnea testing, physicians can abort the test or fail to confirm brain death if severe hemodynamic instability and respiratory abnormalities such as hypoxia continued in potential brain dead donors without signs of improvement. Aborted apnea test means that a determination of brain death is not made and that organ donation and procurement may not take place. As the demand for new organs for transplants is growing more rapidly than the supply, clinical efforts are needed to increase successful completion of apnea tests and organ donation rates by focusing on identifying risk factors associated with apnea test failure and providing aggressive risk factor management. In 2010, Yee et al.[5] claimed in their retrospective study involving 207 potential brain dead donors that the risk of apnea test aborted or failed increased in young patients and when patients showed high a-a gradients or acidemia according to the results of standard ABG test conducted before apnea testing. In this study, the a-a gradient was higher in the failure group showing a significantly increased mean value, compared with that of the success group. Elevated a-a gradient was also identified as a risk factor significantly correlated to apnea test failure in multivariate analysis.

The a-a gradient is a measure of the difference in oxygen concentration between alveolus and artery and useful to identify a cause of intrapulmonary or extrapulmonary hypoxia. Hypoxia that occurs when a-a gradient is normal may be caused by the lack of $\mathrm{F}_{\mathrm{iO} 2}$ and increased $\mathrm{P}_{\mathrm{aCO} 2}$ following central hypoventilation. However, increased a-a gradient suggests hypoxia caused by intrapulmonary ventilation-perfusion (V/Q) imbalance or shunting. In an effort to prevent hypoxia-induced complications and organ damage during apnea testing, preoxygenation is usually performed for 10 minutes. Preoxygenation, if adequately performed, can remove alveolar nitrogen and facilitate the exchange of oxygen. Inadequate preoxygenation however may increase the amount of alveolar nitrogen and cause inadequate exchange of oxygen. Therefore, V/Q mismatch can easily occur, increasing the risk of hypoxia during apnea testing.[8] That is, V/ $\mathrm{Q}$ mismatch reduces resistance to changes in respiratory function during apnea testing and increases the possibility of aborting the test. The a-a gradient indicates degree of intrapulmonary shunting or V/Q mismatch, and it is likely to provide useful information on risk factors for respiratory system. In addition to brain death, patients with serious brain injury commonly develop acute lung injury as a result of neurological pulmonary edema and secondary inflammatory response.[9] In such a setting, the degree of acute lung injury can be assessed by the $\mathrm{P}_{\mathrm{aO} 2} /$ 
$\mathrm{F}_{\mathrm{iO} 2}$ ratio.[5] Although the $\mathrm{P}_{\mathrm{aO} 2} / \mathrm{F}_{\mathrm{iO} 2}$ ratio was not found to be a significant risk factor in multivariate analysis, it was significantly higher in the success group, compared with the failure group, in this study. The findings of this study therefore confirms the need to perform more adequate preoxygenation and aggressive MV for patients with elevated a-a gradient and reduced $\mathrm{P}_{\mathrm{aO} 2} / \mathrm{F}_{\mathrm{iO} 2}$ in order to prevent further injury or collapse of the lung from occurring when respiratory center function is damaged. Once preoxgenation is complete, potential brain dead donors are removed from MV to begin an apnea test, which then results in deterioration of spontaneous respiratory central function and accumulation of carbon dioxide in the blood. Hypercapnea induces respiratory acidosis in brain dead patients, and severe acidemia expands peripheral blood vessels and reduces contractility and myocardial reactivity and conduction velocity in response to catecholamine, leading to low cardiac output such as hypotension.[10-13] Also, the extensive loss of brain functions can damage the hypothalamic-pituitary-peripheral axes and results in a lack of vasopressin known as antidiuretic hormone. Consequently, central diabetes insipidus occurs and causes excessive discharge of urine, hypernatremia and hypovolemic, leading to dilation of blood vessels and hypotension. Hypotension-induced hemodynamic deterioration is an important cause of failure to complete an apnea test and organ hypoperfusion, although maintenance of end-organ perfusion is critical for the preservation of organs to be donated. In this study, pre-apnea low systolic blood pressure was also found to be a significant risk factor associated with apnea test failure in multivariate analysis. To prevent apnea test failure induced by low systolic blood pressure, the administration of intravenous fluids, vasoconstrictors such as vasopressins and vasopressors such as catecholamine and dopamine is recommended. While vital signs are monitored closely, these medications can be administered in a timely manner.[14]

In Korea, this study is the first to identify risk factors for apnea test failure. The findings of this study along with analysis strategies used, provide meaningful insights. However this study has limitations. We could not deter- mine cut-off value of each risk factor required to compile guidelines for pre-apnea treatment because it was not possible to identify effective cut off values with small sample size. To overcome this limitation, further large scale studies are needed to determine cut-off value of each risk factor for apnea test failure and prepare detailed guidelines for pre-apnea treatment.

In conclusion, we identified pre-apnea low systolic blood pressure and elevated a-a gradient (based on ABGA results) as independent risk factors for apnea test failure in potential brain dead donors. Given growing imbalance between supply and demand for organs for transplants, it is important than ever to modify these risk factors aggressively in an effort to reduce failure rates of apnea test and improve organ procurement.

\section{References}

1) Scott JB, Gentile MA, Bennett $S N$, Couture $M$, MacIntyre NR: Apnea testing during brain death assessment: a review of clinical practice and published literature. Respir Care 2013; 58: 532-8.

2) Practice parameters for determining brain death in adults (summary statement). The Quality Standards Subcommittee of the American Academy of Neurology. Neurology 1995; 45: 1012-4.

3) Saposnik G, Rizzo G, Deluca JL: Pneumothorax and pneumoperitoneum during the apnea test: how safe is this procedure? Arq Neuropsiquiatr 2000; 58: $905-$ 8.

4) Bar-Joseph G, Bar-Lavie Y, Zonis Z: Tension pneumothorax during apnea testing for the determination of brain death. Anesthesiology 1998; 89: 1250-1.

5) Yee AH, Mandrekar J, Rabinstein AA, Wijdicks EF: Predictors of apnea test failure during brain death determination. Neurocritical Care 2010; 12: 352-5.

6) Mellemgaard K: The alveolar-arterial oxygen difference: its size and components in normal man. Acta physiol Scand 1966; 67: 10-20.

7) van der Jagt M, Lin MS, Briegel J: Optimizing apnea 
testing to determine brain death. Intensive Care Med 2016; 42: 117-8.

8) Goudreau JL, Wijdicks EF, Emery SF: Complications during apnea testing in the determination of brain death: predisposing factors. Neurology 2000; 55: 1045-8.

9) Avlonitis VS, Wigfield CH, Kirby JA, Dark JH: The hemodynamic mechanisms of lung injury and systemic inflammatory response following brain death in the transplant donor. Am J Transplant 2005; 5: 684-93.

10) Andersen MN, Border JR, Mouritzen CV: Acidosis, catecholamines and cardiovascular dynamics: when does acidosis require correction? Ann Surg 1967; 166: 344-56.
11) Cingolani HE, Faulkner SL, Mattiazzi AR, Bender HW, Graham TP Jr: Depression of human myocardial contractility with "respiratory" and "metabolic" acidosis. Surgery 1975; 77: 427-32.

12) Orchard CH, Kentish JC: Effects of changes of $\mathrm{pH}$ on the contractile function of cardiac muscle. Am J Physiol 1990; 258: C967-81.

13) Kim SY, Koh SO, Yoo YC, Kim HK, Yun TJ, Chang EJ, et al: Successful heart transplantation after dobutamine, glucose-insulin-potassium, and hormone therapy in a hemodynamically unstable cadaveric heart donor. Korean J Crit Care Med 2010; 25: 89-92.

14) Jeret JS, Benjamin JL: Risk of hypotension during apnea testing. Arch Neurol 1994; 51: 595-9. 